The Nitride Workshop is the 8th in a series that began in St. Louis in 1992. Participation of the entire GaN materials and device communities is encouraged, with subject areas including: substrate development • epitaxial growth • properties and applications of narrow-gap III nitrides • structural characterization • optical and electrical characterization - defects, doping and compensation • polarity • alloys and heterostructures - nanostructures • etching • UV optical sources and detectors • optoelectronic devices • III-nitride electronic devices.

\title{
Field of Dreams
}

Truth or dare: How many of you have described the job of a materials scientist as inventing (or developing, designing, or creating) new materials to enable new technologies?

Materials scientists very often respond to the need for a material with a particular set of properties-usually by adapting the processing or composition of an existing material to meet the new need-but it is very rare to invent entirely new materials for which there is no established application. Even when new materials are developed, it is as likely to happen by divine providence as it is by the design process. Stainless steel, Teflon, nylon-all were discovered by accident.

If you do invent
something new, there is
only a small chance that it
will ever be commercialized
and even less that it will
make you rich.

The first plain fact of the matter is that it is really difficult to invent new materials. Those of you with access to large numbers of fertile young minds might want to try this classroom exercise: Ask your students what novel set of properties they would like to design into a new material. The answers will probably disappoint you. You will certainly get a few "superstrong, ultralight" answers, although they never specify how strong or how light. You may get a handful of "transparent alu- minum" responses from the Star Trek fans. If you have done a good job of educating your students, some of them might respond with "ductile ceramics," and that is about the limit of the imagination that you can really expect. The more hopeful educators out there will already have considered the follow-up questions: "What application would your materials have?" and "How would you achieve the specified properties?" But you are not very likely to get that far based on the typical response patterns to the first question.

There are some interesting examples of invented materials. Polyethylene was developed by ICI just before the second world war and was used to provide insulation for the electrical wiring in radar systems, improving performance such that the Allies could use airborne radar to detect the German air force. The world production of polyethylene (by weight) is now the greatest of any synthetic material, and its applications, although initially undreamed of, are legion. Silicon (along with the technologies for doping it) have had a profound influence on society that I hardly need to describe here. Data storage was revolutionized by giant magnetoresistance, then colossal magnetoresistance. And, who knows what is next-humungous magnetoresistance? (There is clearly a need for a SI-sanctioned progression in this kind of terminology, paralleling kilo-, mega-, giga-, and so on.)

The second simple fact is that if you do invent something new, there is only a small chance that it will ever be commercialized and even less that it will make you rich. High-temperature superconductors were supposed to give us new super-efficient cars, but commercial applications have been slow to come so far (although the next generation of cruise ships may be powered by superconducting motors, and some of us have some nice superconducting quantum interference device magnetometers). Buckyballs and nanotubes are truly beautiful materials, but they were discovered rather than designed, and again their applications are more notional than real at this point. Someone will find something to do with these things, I am sure, but it will be a while before they appear in any household products the way polyethylene does.

The best way to a commercial product is to design (rather than invent or discover) a material that meets an established set of needs-negligible thermal expansion, negative thermal expansion, negative Poisson ratio, low-dielectric constant, high-dielectric constant. If you can make a material with properties like these, it might make you rich, but the critical step will be persuading a product manufacturer to adopt it. The sad reality is that adopting new materials in consumer products presents enormous risks-technical, commercial, and legal-and it is the mass markets that provide us with realistic opportunities for great wealth. Despite all these hurdles, meeting existing needs is a much more reliable path to wealth than inventing a solution for a problem that is yet to occur.

But go ahead and dream: If you build it, they will come. They may just take a while getting there.

Alex KING 